Rev. Elev. Méd. vét. Pays trop., 1979, 32 (1) : 11-17

\title{
Le Rafoxanide (3,5-Diiodo-3'-Chloro-4' (P-Chloro-Phenoxy)-Salicylanilide) dans la lutte contre les distomes des ruminants domestiques en Afrique tropicale
}

\author{
par M. GRABER $\left(^{*}\right)$
}

\begin{abstract}
RÉSUMÉ
En Afrique tropicale, le Rafoxanide peut être utilisé, chez les ruminants domestiques, pour le traitement des distomatoses hépato-biliaires à Fasciola hepatica et à Fasciola gigantica, de certaines "Strongyloses» gastro-intestinales et de l'Oestrose à Oestrus ovis.

Il semble dépourvu d'efficacité à l'égard des Paramphistomes adultes de a panse. Toutefois, chez le mouton, il ferait preuve, à forte dose, d'une grande efficacité vis-à-vis des formes immatures implantées dans la muqueuse duodénale.

Chez le zébu, lors de distomatose chronique (douves de plus de huit semaines) et de strongylose, le médicament peut être injecté par la voie sous-cutanée en solution à 5 ou 7,5 p. 100 . La dose sera, au minimum, de $3 \mathrm{mg} / \mathrm{kg}$. Lors de distomatose aigue (douves de moins de huit semaines), elle sera comprise entre 5 et $7,5 \mathrm{mg} / \mathrm{kg}$.

Administrées par la voie buccale (suspension à 2,5 p. 100), les doses représentent environ le double des précédentes, c'est-à-dire :

- Distomatose chronique, strongyloses gastro-intestinales, cestrose ovine : $7,5 \mathrm{mg} / \mathrm{kg}$ chez tous les Ruminants domestiques. les bovins.

- Distomatose aíguè : $10 \mathrm{mg} / \mathrm{kg}$ chez les ovins ; de 10 à $15 \mathrm{mg} / \mathrm{kg}$ chez

L'emploi et la posologie du Rafoxanide devront tenir le plus grand compte de l'épidémiologe de la distomatose en Afrique.
\end{abstract}

Le Rafoxanide ou 3,5-diiodo-3'-chloro-4'(p-chloro-phénoxy)-salicylanilide (**) est un médicament connu depuis une dizaine d'années (10) pour son activité à l'égard des douves hépatobiliaires et, à ce titre, il est actuellement très utilisé chez les ruminants domestiques dans la lutte contre les maladies provoquées par ces distomes.

En Afrique, il a fait l'objet de nombreuses recherches en Angola (5), en Afrique du Sud $(8,9,12,13,14,15,16,17)$, ̀̀ Madagascar (3, 4,11 ), au Tchad (18) et au Sénégal (1). Ses indications ont été bien précisées et le Ranide s'est révélé efficace non seulement vis-à-vis des
Fasciola, mais également de certains Nématodes des bovins et des ovins.

Aussi, en raison de l'intérêt que présente cet anthelminthique du fait de sa polyvalence, a-t-il paru utile, à la lumière des observations faites ces dernières années, de donner quelques renseignements sur ses indications, sa posologie et son utilisation en Afrique tropicale.

(*) Chaire de Parasitologie. Ecole Nationale Vétérinaire de Lyon. Marcy l'Etoile, 69260 Charbonnières-LesBains.

(**) Commercialisé sous les noms déposés de Flukanide et de Ranide. 


\section{LE MÉDICAMENT}

Le Rafoxanide se présente sous l'aspect d'une poudre de couleur blanche tirant sur le gris jaune, renfermant 98 p. 100 de principe actif.

En thérapeutique, il est utilisé sous deux formes :

- En suspension à 2,5 p. 100 colorée en jaune clair et s'administrant directement par voie orale,

- En solution à 5 et 7,5 p. 100 injectable par la voie sous-cutanée.

\section{INDICATIONS ET POSOLOGIE}

\section{Rafoxanide en suspension}

\subsection{Traitement des distomatoses hépato-bi- liaires}

Elles sont dues en Afrique à la présence dans le foie des ruminants de deux Trématodes : Fasciola hepatica qui colonise l'Afrique du Nord, certaines régions d'Afrique du Sud, ainsi que les hautes terres de l'Est africain (Kenya, Ethiopie) et Fasciola gigantica que l'on trouve partout ailleurs.

Les douves adultes se localisent dans les canaux biliaires et les douves immatures en cours de migration, dans le parenchyme hépatique. Dans le premier cas, la maladie qu'elles provoquent prend une allure chronique et, dans le second, une allure aiguẽ. Classiquement, on admet que la distomatose chronique est le fait du bcuf et du zébu, tandis que la distomatose aiguë se voit surtout chez le mouton. En Afrique, cette distinction n'est pas toujours aussi nette et il n'est pas exceptionnel d'observer chez les bovins des cas de fasciolose massive, grave et à évolution rapide, surtout lorsque Fasciola hepatica et Fasciola gigantica sont associées, comme c'est le cas dans certains pays (Ethiopie).

Dans les deux espèces, la phase aiguë se situe entre la quatrième et la huitième semaine faisant suite à l'ingestion des métacercaires infestantes et la phase chronique au-delà de la huitième semaine.

La durée de la phase prépatente est de 70 jours environ pour Fasciola hepatica et de 92-98 jours pour Fasciola gigantica.

La posologie du Rafoxanide varie selon les espèces animales et l'âge des parasites en cause.

\subsubsection{Fasciola hepatica}

- Chez le mouton, suivant les expérimentateurs et les techniques employées, les pourcentages d'efficacité à l'égard des douves immatures sont assez dissemblables :

- Douves âgées de 4 semaines : 52 , 91 et $92 \mathrm{p} .100$ à $5,7,5$ et $10 \mathrm{mg} / \mathrm{kg}(7) ; 46 \mathrm{p}, 100$ à $7,5 \cdot \mathrm{mg} / \mathrm{kg}(15)$.

- Douves âgées de 5 semaines : 54 p. 100 à $7,5 \mathrm{mg} / \mathrm{kg}(15)$.

- Douves âgées de 6 semaines : 89,92 et 97 p. 100 à $5,7,5$ et $10 \mathrm{mg} / \mathrm{kg}(7) ; 81$ à 89 p. 100 à $7,5 \mathrm{mg} / \mathrm{kg}(9,15) ; 94$ p. 100 à $7 \mathrm{mg} / \mathrm{kg}(2)$.

La dose recommandée pour le traitement de la distomatose aiguë du mouton se situe donc autour de $10 \mathrm{mg} / \mathrm{kg}$.

En ce qui concerne la distomatose chronique, la dose retenue en Europe est de $7,5 \mathrm{mg} / \mathrm{kg}$. Elle est également valable en Afrique, bien que des posologies nettement inférieures ne soient pas dépourvues de toute activité à l'égard de ce parasite (2).

- Chez le bœuf et chez le zébu (17), lors d'infestations naturelles, sur des douves âgées de plus de 100 jours, le pourcentage de douves tuées à $3,75 \mathrm{mg} / \mathrm{kg}$ est, en moyenne, de 64 p. 100 (de 49 à 66 p. 100), à $5 \mathrm{mg} / \mathrm{kg}$ de 92 p. 100 (de 66 à $95 \mathrm{p} .100$ ) et à $7,5 \mathrm{mg} / \mathrm{kg}$, de $100 \mathrm{p} .100$.

Les douves de 6 semaines sont beaucoup moins sensibles : 36 et 56 p. 100 à 7,5 et $10 \mathrm{mg} / \mathrm{kg}$. Il faut donc prévoir, dans ce cas, une posologie plus élevée.

\subsubsection{Fasciola gigantica}

- Chez le mouton, les douves âgées de 75 à 100 jours sont, en grande partie, éliminées à 7 et $7,5 \mathrm{mg} / \mathrm{kg}$ : plus de $80 \mathrm{p} .100(9) ; 100 \mathrm{p} .100$ $(2,15)$. A $5 \mathrm{mg} / \mathrm{kg}$, elles le sont dans la proportion de 82 à 95 p. $100(9,15)$.

Sur les douves immatures, les résultats sont moins favorables. Les pourcentages de réduction sont, à $7,5 \mathrm{mg} / \mathrm{kg}$, de $90 \mathrm{p}$. 100 pour des douves de 50 jours (15) et, à $7 \mathrm{mg} / \mathrm{kg}$, de $50 \mathrm{p}$. 100 pour des douves de 6 semaines (2). Les fascioles de 25 jours sont encore plus résistantes et une dose de $15 \mathrm{mg} / \mathrm{kg}$ ne parvient à chasser que les deux tiers des trématodes (9).

- Chez le bouf et chez le zébu, l'activité à l'égard des Fasciola gigantica adultes se manifeste dès $2,5 \mathrm{mg} / \mathrm{kg}(13,14,18)$. A $3,75 \mathrm{mg} / \mathrm{kg}$, quelques fascioles demeurent vivantes $(13,14,15)$ ainsi qu'à $5 \mathrm{mg} / \mathrm{kg}(3,13,15)$. 
A $7,5 \mathrm{mg} / \mathrm{kg}$, les auteurs sont tous d'accord, pour reconnaître que le médicament est efficace à 100 p. $100(1,3,14,15,18)$.

Les immatures sont - comme chez le mouton - plus difficiles à atteindre (1) et les pourcentages de réduction varient sensiblement selon les auteurs et l'âge des parasites :

- Sur des douves âgées de 6 semaines: à 7,5 et $10 \mathrm{mg} / \mathrm{kg}, 38$ et 56 p. $100(15)$;

- Sur des douves âgées de 8 semaines : à 5 et $7,5 \mathrm{mg} / \mathrm{kg}, 37-48$ et 75 p. $100(14,18)$; à 10,11 , 2,15 et $20 \mathrm{mg} / \mathrm{kg}, 86-96,72,5,92-99$ et $98-100$ p. $100(3,14,15,18)$.

1.1.3. En définitive, chez le mouton, le traitement des distomatoses à Fasciola hepatica ou à Fasciola gigantica nécessite, en Afrique, une posologie de $7,5 \mathrm{mg} / \mathrm{kg}$ lorsqu'il s'agit de distomatose chronique et de $10 \mathrm{mg} / \mathrm{kg}$ au moins, lorsque la fasciolose est aiguë.

Chez le bœuf et chez le zébu, les distomes adultes sont détruits à $7,5 \mathrm{mg} / \mathrm{kg}$. Par contre, l'élimination des douves de moins de 8 semaines dont la croissance dans cette espèce est plus lente que dans l'espèce ovine (17) exige une dose plus forte comprise entre 10 et $15 \mathrm{mg} / \mathrm{kg}$. Le respect de cette posologie est indispensable lorsque l'on ne connaît pas exactement l'âge des douves ou lors d'infestation massive avec formation sur le trajet des gros canaux biliaires de poches plus ou moins volumineuses où s'accumulent les parasites que le médicament à dose normale $(7,5 \mathrm{mg} / \mathrm{kg})$ n'arrive pas à atteindre.

\subsection{Autres indications}

\subsubsection{Traitement de la paramphistomose}

Elle se manifeste sous deux aspects :

- Une forme aiguë due à la localisation dans le_duodénum de jeunes paramphistomes immatures qui y séjournent un mois environ ;

- Passé ce temps, ils effectuent une migration rétrograde qui les mène dans le rumen où ils deviennent adultes. La paramphistomose prend alors une allure chronique.

Chez le mouton, le Rafoxanide à $15 \mathrm{mg} / \mathrm{kg}$ chasse 93 p. 100 des trématodes présents (Paramphistomum microbothrium) dans les premières parties de l'intestin grêle (9).

En matière de paramphistomose chronique, les renseignements sont rares. La seule observation que l'on possède est celle de BOUCHET, DAYNES et BIRGI (3) à Madagascar : aux doses utilisées chez le zébu $(5,7,515$ et $20 \mathrm{mg} / \mathrm{kg})$, le médicament ne semble pas actif vis-à-vis des paramphistomes de la panse (Paramphistomum et Carmyerius).

Et cependant, en Afrique tropicale, ces affections ne doivent pas être négligées. Le rôle pathogène des Paramphistomum et des Carmyerius, tant au stade larvaire qu'au stade adulte, est indéniable. Au cours des dernières années où un grand nombre de pays africains ont subi les effets d'une sécheresse prolongée, des accidents mortels, voire de véritables enzooties, ont été observés chez des moutons et des bovins utilisant les rares pâturages encore disponibles, c'est-à-dıre des zones marécageuses particulièrement favorables à l'évolution de ces trématodes (Nigeria, Ethiopie).

Par ailleurs, les Paramphistomes sont très souvent associés aux Fasciola, car leurs hôtes intermédiaires (Limnées et Bulins) cohabitent fréquemment dans le même milieu. On a donc intérêt à sélectionner le plus possible des anthelminthiques actifs à la fois sur les Fasciola et sur les paramphistomatidés.

\section{I.2.2. Traitement de certaines « strongyloses》 gastro-intestinales}

- Chez le mouton, le Rafoxanide à $7,5 \mathrm{mg} / \mathrm{kg}$, fait preuve d'une bonne activité à l'égard d'Haemonchus contortus $\left(^{*}\right)$ et de Gaigeria pachyscelis à tous les stades d'évolution. Sur Chabertia ovina, le pourcentage d'efficacité dépasse 85 p. $100(9,16)$.

- Chez les bovins (17), toujours à $7,5 \mathrm{mg} / \mathrm{kg}$, le Rafoxanide élimine 95 p. 100 des Bunostomum phlebotomum adultes et 87 p. 100 des Haemonchus place $i$ âgés de 3 à 4 jours.

\section{I.2.3. Traitement de l'astrose à Oestrus ovis.}

Le médicament est excrété dans le mucus nasal (12). Il entre en contact avec les larves d'œstres (stades 1, 2 et 3 ) et les tue si la concentration est suffisante, ce qui est le cas à $7,5 \mathrm{mg} / \mathrm{kg}(4,8,16)$.

\section{Rafoxanide en solution injectable}

Chez le zébu, des essais effectués en Afrique du Sud (12) ont montré que le Rafoxanide en solution à 5 p. 100 injectée par la voie souscutanée faisait preuve d'une bonne activité à l'égard des Fasciola hepato-biliaires et de plusieurs nématodes de la caillette et de l'intestin, activité que l'on peut résumer ainsi :

(*) Sauf sur les larves $L_{4}$ en état d'hypobiose. 
Fasciola hepatica adultes

Fasciola gigantica adultes

Paramphistomum microbothrium immatures

Bunostomum phlebotomum adultes

Haemonchus placei adultes

Haemonchus placei de 1-2 jours

Bosicola radiatum adultes

Larves $\mathrm{L}_{4}$ de Bosicola radiatum

Fasciola gigantica âgées de 56 jours

Haemonchus placei de 1-2 jours

Fasciola gigantica âgées de 42 jours

\begin{tabular}{|c|c|c|}
\hline Dose & $\begin{array}{l}\text { Pourcentage } \\
\text { de réduction }\end{array}$ & \begin{tabular}{|} 
Nombre d'animaux \\
totalement déparasités
\end{tabular} \\
\hline $3 \mathrm{mg} / \mathrm{kg}$ & $\begin{array}{l}83 \text { p. } 100 \\
99 \text { p. } 100 \\
10 \text { p. } 100 \\
99 \text { p. } 100 \\
99 \text { p. } 100 \\
73 \text { p. } 100 \\
99 \text { p. } 100 \\
77 \text { p. } 100\end{array}$ & $\begin{array}{r}2 \text { sur } 11 \\
10 \text { sur } 11 \\
0 \text { sur } 11 \\
10 \text { sur } 11 \\
7 \text { sur } 11 \\
1 \text { sur } 11 \\
10 \text { sur } 11 \\
0 \text { sur } 11\end{array}$ \\
\hline \multirow{2}{*}{$7,5 \mathrm{mg} / \mathrm{kg}$} & $\begin{array}{l}97 \text { p. } 100 \\
99 \text { p. } 100\end{array}$ & $\begin{array}{l}4 \text { sur } 11 \\
2 \text { sur } 11\end{array}$ \\
\hline & 92 p. 100 & 1 sur 11 \\
\hline
\end{tabular}

A Madagascar (11), chez des zébus atteints de distomatose chronique à Fasciola gigantica, des doses de $1,1,5$ et $2 \mathrm{mg} / \mathrm{kg}$ ont été expérimentées. Le pourcentage de réduction du nombre de parasites est de 96,8 p. 100 dans le premier cas, de 98,1 p. 100 dans le second et de 100 p. 100 dans le troisième. Il reste un certain nombre d'animaux porteurs de Fasciola à $1 \mathrm{mg} / \mathrm{kg}$ (2 sur 5 ) et $1,5 \mathrm{mg} / \mathrm{kg}$ ( 1 sur 5 ).

Il est difficile de comparer 2 expériences effectuées dans des conditions différentes: infestations naturelles à Madagascar, infestations expérimentales en Afrique du Sud. Par ailleurs, avec le Rafoxanide administré par la voie buccale, on sait qu'à partir de doses faibles $(2,5$ et $3,75 \mathrm{mg} / \mathrm{kg})$, certains auteurs obtiennent d'excellents résultats qui ne sont pas entièrement confirmés par d'autres, l'unanimité se faisant à $7,5 \mathrm{mg} / \mathrm{kg}$ (voir supra). Interviennent vraisemblablement l'étendue et l'état des lésions, l'âge des parasites, les infestations naturelles anciennes étant, en général, plus faciles à détruire que les infestations plus récentes.

Avec le Rafoxanide injectable, on peut considérer que $3 \mathrm{mg} / \mathrm{kg}$ (12) est une dose limite inférieure qui assure l'élimination de la plus grande partie des Fasciola gigantica âgées de 98 jours. Cependant, quelques animaux demeurent porteurs d'un petit nombre de parasites mûrs capables de disséminer ultérieurement la maladie.

Dans ces conditions - et sous réserve de confirmations par d'autres essais -, il est raisonnable de penser que les doses de Rafoxanide injectable représentent la moitié -- ou peut-être un peu moins (12) - des doses de Rafoxanide buvable. Cette opinion est corroborée par le fait que, pour les douves de moins de huit semaines, les doses recommandées par SCHRÖDER, HONER et LOUW (12) sont de
5 et de $7,5 \mathrm{mg} / \mathrm{kg}$, ce qui correspond à la moitié des doses de Rafoxanide buvable (10 et $15 \mathrm{mg} / \mathrm{kg}$ ) retenues pour le traitement de ce type de distomatose (Voir supra).

\section{UTILISATION DU RAFOXANIDE EN AFRIQUE TROPICALE}

En matière de distomatose, deux interventions sont souhaitables (6) que l'on peut, en Afrique Soudano-sahélienne, schématiser ainsi :

- La première tactique : de février à juin, selon les latitudes. Elle est destinée à tuer le plus grand nombre possible de jeunes fascioles acquises durant cette période qui, dans l'hémisphère Nord, couvre le milieu et la fin de la saison sèche. A cette époque où les ressources fourragères diminuent notablement aboutissant à une situation de disette ou de sub-disette alimentaire, ce traitement ne peut être que bénéfique. Il aidera les animaux à mieux franchir le cap de la soudure alimentaire et réduira les pertes de poids qui, en Afrique centrale, chez certains zébus de boucherie, peuvent atteindre 25 p. 100 .

Comme il s'agit, la plupart du temps, de douves immatures d'âge indéterminé ou de douveś mûres mélangées à des douves immatures en migration dans le parenchyme hépatique, la dose $\left(^{*}\right)$ à administrer oralement devra être forte : $10 \mathrm{mg} / \mathrm{kg}$ chez ovins et entre 10 et $15 \mathrm{mg} / \mathrm{kg}$ chez les bovins. La dose de $5 \mathrm{mg} / \mathrm{kg}$ indiquée précédemment (18) est manifestement trop faible, surtout lorsque l'on intervient en fin de saison sèche sur des troupeaux transhumants remontant vers le Nord, troupeaux avec lesquels

(*) Avec le Rafoxanide injectable, la dose sera réduite d'environ la moitié. 
le contact est difficile à établir et, la plupart du temps, épisodique. Lorsque l'on y parvient, on a tout intérêt à adopter une posologie élevée permettant de détruire le plus grand nombre possible de trématodes.

- La seconde stratégique, à la fin des pluies, en septembre-octobre. Elle a pour but d'éliminer les douves non touchées par le traitement précédent. Comme à cette époque de l'année, les parasites ont atteint leur maturité sexuelle, une dose de $7,5 \mathrm{mg} / \mathrm{kg}$ suffit.

Pour les animaux d'embouche ou pour ceux qui sont élevés sur des Ranches, par souci de rentabilité, il faut limiter au maximum l'impact de la distomatose et éviter à tout prix des pertes de poids et des retards de croissance. Les doses à administrer seront donc élevées (mouton, $10 \mathrm{mg} / \mathrm{kg}$; bovins-zébus, 10 à $15 \mathrm{mg} / \mathrm{kg}$ ) et répétées - tout au moins dans un premier temps - tous les trois mois, c'est-à-dire avant que les quelques douves survivantes ne deviennent adultes, donc susceptibles de disséminer l'affection et d'en maintenir la pérennité. Ultérieurement, le nombre de traitements annuels pourra être réduit à deux, puis à un seul.

Les animaux nouvellement achetés devront être systématiquement traités à doses élevées avant d'être introduits dans les ranches.

En Ethiopie où coexistent Fasciola hepatica et Fasciola gigantica dont les hôtes et la biologie sont différents, l'intervention tactique à dose élevée aura lieu de septembre à décembre à une époque où Fasciola hepatica fait sentir ses effets et l'intervention stratégique à dose normale $(7,5 \mathrm{mg} / \mathrm{kg})$ en mars-avril, au début des premières pluies.

\section{CONCLUSIONS}

1. Le Rafoxanide fait preuve d'une bonne activité à l'égard de Fasciola hepatica et de Fasciola gigantica, d'Oestrus ovis et de divers nématodes gastro-intestinaux : Haemonchus contortus et Haemonchus placei à tous les stades d'évolution (sauf les larves $\mathrm{L}_{4}$ d'Haemonchus contortus en état d'hypobiose), Gaigeria pachyscelis, Bunostomum phlebotomum, Chabertia ovina et Bosicola radiatum adultes.

Sur les paramphistomes, le médicament à forte dose assure, chez le mouton, la destruction de la plus grande partie des trématodes immatures présents dans le duodénum, tandis que, chez le zébu, il semble dépourvu d'efficacité vis-à-vis des amphistomes adultes implantés dans le rumen.

2. Le médicament s'administre par la voie sous-cutanée, en solution à 5 ou à 7,5 p. 100 ou par la voie buccale en suspension à 2,5 p. 100 .

2.1. Dans le premier cas, chez les bovins, la dose recommandée dans le traitement de la distomatose chronique (douves âgées de plus de 8 semaines) est, au minimum, de $3 \mathrm{mg} / \mathrm{kg}$. Elle se montre active également à l'égard de plusieurs nématodes gastro-intestinaux.

Sur les douves âgées de moins de 8 semaines (distomatose aiguë), elle sera portée à 5$7,5 \mathrm{mg} / \mathrm{kg}$.

2.2. Dans le second cas, les doses préconisées représentent environ le double des précédentes, soit :

Distomatose chronique, strongyloses gastro-intestinales, œstrose ovine : $7,5 \mathrm{mg} / \mathrm{kg}$ chez tous les ruminants domestiques.

— Distomatose aiguë : $10 \mathrm{mg} / \mathrm{kg}$ chez les ovins; de 10 à $15 \mathrm{mg} / \mathrm{kg}$ chez les bovins.

3. La posologie à adopter en Afrique tropicale devra tenir compte de la biologie et de l'évolution des parasites (trématodes et nématodes). Dans les zones soudano-sahéliennes, les traitements effectués en fin de saison sèche, à une époque où les douves sont encore immatures, le seront à des doses élevées, tandis que les interventions de fin de saison des pluies sur des distomes devenus adultes le seront à dose normale.

Dans les ranches et chez les animaux d'embouche, le souci de rentabilité prime tout: il faut alors agir avec la plus grande efficacité, c'est-à-dire à forte dose.

\section{SUMMARY}

\section{Treatment, in tropical Africa, of sheep and cattle Fascioliasis with} Rafoxanide

In field infestation of cattle and sheep, Rafoxanide is indicated in the treatment of fascioliasis (Fasciola hepatica and Fasciola gigantica), of some gastrointestinal «Strongylosis » and of Oestrus ovis. It seems to be ineffective against 
adult stomach flukes (Paramphistomum sp.). In sheep, however, the medicament at higher dose would be active on immature ones in the duodenum.

In chronic fascioliasis (flukes more than eight weeks old) and certain «strongylosis» of zebu cattle, the dosage rate injected subcutaneously in solution at 5 or $7,5 \mathrm{p}$. cent is, to a minimum, $3 \mathrm{mg} / \mathrm{kg}$. In acute fascioliasis (flukes less than eight weeks old), it is $5-7,5 \mathrm{mg} / \mathrm{kg}$. Administered orally, these doses are about twice as high, i. e. :

- Chronic fascioliasis, gastro-intestinal «strongylosis » and nasal bot : $7,5 \mathrm{mg} / \mathrm{kg}$ in all domestic ruminants;

- Acute fascioliasis : $10 \mathrm{mg} / \mathrm{kg}$ in sheep and $10-15 \mathrm{mg} / \mathrm{kg}$ in cattle.

For using Rafoxanide in Africa, the author emphasizes the necessity of carefully taking into account the epidemiology of fascioliasis.

\section{RESUMEN}

\section{El Rafoxanido (3,5-diiodo-3'-cloro-4' (P-cloro-fenoxi) Salicylanilide) en la lucha contra los distomas de los rumiantes domésticos en Africa tropical}

En Africa tropical, se puede utilizar el Rafoxanido, en los rumiantes domésticos, para el tratamiento de las distomatosis hepatobiliares con Fasciola hepatica y Fasciola gigantica, de algunas estrongilosis gastrointestinales y de la infección causada por Oestrus ovis.

Parece privado de eficacidad para con los Paramfistomos adultos de la panza. Sin embargo, en la oveja, manifiesta, en dosis elevada, una gran eficacia con respecto a las formas inmaduras establecidas en la mucosa duodenal.

En el cebú, durante distomatosis crónica (distomas hepáticos de más ocho semanas y estrongilosis, se puede inyectar el medicamento por vía subcutánea en solución a 5 o 7,5 p. 100 . La dosis sera al minimo de $3 \mathrm{mg} / \mathrm{kg}$. Durante distomatosis aguda (distomas de menos de ocho semanas) sera incluida entre 5 y $7,5 \mathrm{mg} / \mathrm{kg}$.

Administradas por via oral (suspensión a 2,5 p. 100), las dosis representan cerca del doble de lás precedentes, es decir :

- distomatosis crónica, estrongilosis gastrointestinales; infección causada por Oestrus ovis : $7,5 \mathrm{mg} / \mathrm{kg}$ en todos los rumiantes domésticos; bovinos.

— distomatosis aguda : $10 \mathrm{mg} / \mathrm{kg}$ en los ovinos; 10 a $15 \mathrm{mg} / \mathrm{kg}$ en $\mathrm{los}$

El empleo y la posología del Rafoxanido deberan tomar en cuenta la epidemiologia de la distomatosis en Africa.

\section{BIBLIOGRAPHIE}

1. BERTHE (J.). Contribution à l'étude du traitement de la fasciolose au Sénégal. Thẻse Méd. vét. Toulouse, 1972, no 61, 74 p.

2. BORAY (J, C.), WOLFF (K.), TREPP (H. C.). Prüfung neuer Faszioliside. I. - W1rksamkeit und Toxizität von Rafoxamıd bei Küstlich mit Fasciola hepatica oder Fasciola gigantica infizierten Schafen. Schweizer Arch. Tierheilk., 1973, 115 (8) : 367-371.

3. BOUCHET (A.), DAYNES (P.), BIRGI (E.). Etude de l'action anthelminthıque d'un dérivé halogéné de la Salicylanilide vis-à-vis de Fasciola gigantica. Rev. Elev. Méd. vét. Pays trop., 1971, 24 (4) : 543-549.

4. BOUCHET (A.), DUPRE (J. J.), RAKOTOZANANY (E.). Traitement de l'œestrose ovine. II. Essais réalisés avec le Rafoxanide. Revue Elev. Méd. vét. Pays trop., 1974, 27 (3) : 281-284.

5. CRUZ e SILVA (J. A.) et collab. L'efficacité du Rafoxanide dans la fasciolose à Fasciola gigantica. Vet. Moçamb., Lourenco Marques, 1973, 6 (2) : 79.

6. GRABER (M.). Les trématodes hépatiques et gastriques d'Afrique centrale. Rev. Elev. Méd. vét. Pays trop., 1975, 28 (3) : 311-314.

7. GUILHON (J.), JOLIVET (G.), BARNABE (R.). Activité du 3,5-diiodo-3'-chloro-4'-(p-chlorophénoxy) salicylanilide sur les formes immatures de Fasciola hepatica. Bull. Acad. vét. Fr., 1970, 43 (10) : 419-426.

8. HORAK (I. G.), LOUW (J. P.), RAYMOND (S. M.). Trials with Rafoxanide. III. - Efficacy of Rafoxanide against the larvac of sheep nasal bot Oestrus ovis Linné, 1761. Jl. S. Afr. vet. med. Ass., 1971, 42 (4) : 337-339.

9. HORAK (I. G.), SNIJDERS (A. J.), LOUW (J. P.). Trials with Rafoxanide. V. - Efficacy studies against Fasciola hepatca, Fasciola gigantica, Paramphistomum microbothrium and various Nematodes in sheep. $J l . S$. Afr. vet. Ass., 1972,43 (4) : 387-403.

10. MZOZIK (H.) et collab. A new agent for the treatment of liver fluke infection (Fascioliasis). Experientia, 1969, 25 : 883.

11. RAZAFINDRAKOTO (Ch.), RANAIVOSON (A.), MEGARD (J. P.). Efficacité du Rafoxanide injectable contre Fasciola grgantica adulte chez le zébu à Madagascar. Rev. Elev. Méd. vét. Pays trop., 1978, 31 (2) : 165-169.

12. SCHRÓDER (J.), HONER (M. R.), LOUW (J. P.). Trials with Rafoxanide, VIII. - Efficacy of an injectable solution against Trematodes and Nematodes in cattle. Jl. S. Afr. vet. Ass., 1977, 48 (2) : 95-97. 
13. SNIJDERS (A. J.), LOUW (J. P.), SERRANO (F. M. H.), Trials with Rafoxanide. I. - Fasciola gigantica in cattle in Angola. Jl. S. Afr. vet. med. Ass., 1971, 42 (3) : 245-251.

14. SNIJDERS (A. J.), HORAK (I. G.), LOUW (J. P.). Trials with Rafoxan1de. II. - Efficacy against Fasciola gigantica in cattle. Jl. S. Afr. vet. med. Ass., 1971, 42 (3) : 253-257.

15. SNIJDERS (A, J.), HORAK (I, G.). Treatment with Rafoxanide of Fasciola hepatica and Fasciola gigantica in sheep and cattle. 19th Int. Cong. Med. Vet., Mexico, 1971, 2, 760.

16. SNIJDERS (A. J.), HORAK (I. G.), LOUW (J. P.).
Trials with Rafoxanide. VI. - The effect of repeated and single treatments with Rafoxanide against $H a e-$ monchus contortus and Oestrus ovis in sheep. JI. S. Afr. vet. Ass., 1973, 44 (3) : 251-263.

17. SNIJDERS (A. J.), HORAK (I. G.). Trials with Rafoxanide. VII. - Efficacy against Fasciola hepatica, Haemonchus placei and Bunostomum phlebotomum in cattle. Jl. S. Afr. vet. Ass., 1975, 46 (3) : 265-267.

18. TRONCY (P. M.), VASSEAU-MARTIN (N.). Le Rafoxanide dans le traitement de la fasciolose à Fasciola gigantica chez le zébu du Tchad. Rev. Elev. Med. vét. Pays trop., 1976, 29 (1) : 31-37. 